\title{
Trabajo Social y gestión. Oportunidad conflictuada
}

\author{
Raúl Octavio Hozven Valenzuela ${ }^{1,2}$ \\ https://orcid.org/0000-0001-5802-0635 \\ ${ }^{1}$ Universidad Santo Tomás, Facultad de Ciencias Sociales y Comunicaciones, Escuela de Trabajo Social, Viña del Mar, VA, Chile \\ ${ }^{2}$ Pontificia Universidad Católica de Valparaíso, Escuela de Psicología, Programa de Doctorado en Psicología, Viña del Mar, VA, Chile
}

\section{Trabajo Social y gestión. Oportunidad conflictuada}

Resumen: El presente artículo se pregunta respecto a la construcción subjetiva que emerge en la conflictiva relación entre Trabajo Social y la gestión de políticas sociales. Con este fin se realiza un análisis de discurso conforme la perspectiva de los autores Potter y Wetherell, tomando como referencia entrevistas vinculadas a un proyecto de investigación disciplinar desarrollado entre los años 2016 y 2017. Los resultados que emergen en los textos dan cuenta de un discurso de emprendimiento en torno al Trabajo Social, que, después de la recuperación del rango universitario acaecida en Chile en 2005, tensiona las tradiciones de esta profesión ante los mandatos establecidos por el Nuevo Management Publico (NMP), forjando conflictos no solo a escala del trabajo, sino que también culturales.

Palabras clave: Gestión social. Subjetividad. Materialidades. Trabajo Social. Nuevo Management Público (NMP).

\section{Serviço Social e gestão: oportunidade conflituada}

Resumo: Este artigo questiona a construção subjetiva que emerge na relação conflituosa entre o Serviço Social e a gestão de políticas sociais. Para tal, é realizada uma análise do discurso segundo a perspectiva dos autores Potter e Wetherell, tendo como referências entrevistas vinculadas a um projeto de pesquisa disciplinar desenvolvido entre os anos de 2016 e 2017 . Os resultados que emergem nos textos dão conta de um discurso de empreendedorismo em torno do Serviço Social, que, após a recuperação do nível universitário ocorrido no Chile em 2005, acentua as tradições dessa profissão perante os mandatos estabelecidos pela Nova Gerência Pública, forjando conflitos não apenas no âmbito do trabalho, mas também culturais.

Palavras-chave: Gestão social. Subjetividade. Materialidade. Serviço Social. Nova Gerência Pública.

\section{Social Work and management: Conflicting opportunity}

Abstract: This article questions the subjective construction that emerges in the conflicting relationship between Social Work and social policy management. The study analyzes the discourse following the perspective of Potter and Wetherell, using as references interviews applied in a research project developed between 2016 and 2017. The results demonstrate a discourse of entrepreneurship around the field of Social Work, which, after the recovery of the university level that occurred in Chile in 2005, emphasize the traditions of the profession before the mandates established by the New Public Management (NPM), forging cultural and work conflicts.

Keywords: Social management. Subjectivity. Materiality. Social Work. New Public Management (NPM).

Recibido en 13.09.2018. Aprobado en 20.11.2018. Revisado en 08.05.2019.

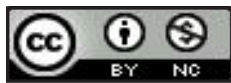

(C) El(Los) Autor(es). 2019 Acceso Abierto Esta obra está licenciada bajo los términos de la Licencia Creative Commons Atribución-NoComercial 4.0 Internacional (https://creativecommons.org/licenses/by-nc/4.0/deed.es), que permite copiar, distribuir y reproducir en cualquier medio, así como también adaptar, transformar y crear a partir de este material, desde que para fines no comerciales, y que usted fornezca el crédito debido a los autores y a la fuente, insiera un enlace para la Licencia Creative Commons e indique si fueron hechas alteraciones. 


\section{Introducción}

En Chile desde 1975 con la dictadura de Pinochet, se comienza a instalar una incesante desregulación laboral, mermando al Estado. Estas transformaciones involucran a las formas de gestión organizacional esbozando el denominado Nuevo Management Público (NMP) (Sisto \& Fardella, 2014), que traslada los modelos existentes en la empresa privada a los servicios públicos, instalándose como un modo de ser en la esfera laboral. En este contexto cobra relevancia abordar la emergencia subjetiva (González-Rey \& Mitjáns, 2016), las normalidades de la normalidad en lo laboral (Grassi, 2009), el disciplinamiento de las fuerzas del trabajo (Vivero Arriagada, 2017) y otros efectos socioculturales. Al respecto, el artículo plantea contribuir a la discusión relacionada con los conflictos que ha acarreado el NMP en las políticas sociales, especialmente a las profesiones que las gestionan, entre las que destaca el Trabajo Social. Por ello, tomando en cuenta a la gestión social como un ámbito de desempeño profesional del Trabajo Social (Castañeda \& Salamé, 2012), el presente artículo traza como propósito analizar la construcción discursiva que emerge en Trabajadores Sociales que gestionan políticas sociales en educación y vivienda, después de la recuperación del rango universitario. La metodología utilizada fue cualitativa, realizándose 23 entrevistas a Trabajadores Sociales, los textos se analizaron en base al análisis de discurso pragmático (Potter \& Wetherell, 1987) en el marco de un proyecto disciplinar, entre los años 2016 y 2017. Con este fin el escrito se estructura en cuatro apartados, en el primero se presenta una breve contextualización del NMP y una historización desde 1980 respecto a materialidades que subjetivan al Trabajo Social en diferentes ámbitos de desempeño. En el segundo de los capítulos se ofrecen contrapuntos conceptuales respecto a la gestión social, incluyendo además una descripción de la técnica de análisis. En la tercera parte se examinan discursos que emanan de las entrevistas de acuerdo a los recursos de factualización (Potter, 1998) que muestran cómo las construcciones de sí se articulan con formas validadas de ser (Sisto \& Fardella, 2008) para finalmente presentar el cierre con las conclusiones.

\section{Un Trabajo Social inscrito "managerialmente"}

El NMP, configura una administración con base en el desempeño individual, aspecto que tracciona el ideario colectivo en la administración pública, inscribiendo al mercado como medio y fin, pero, esta ideología para desplegarse requiere de materialidades que producen modos de actuar (Sisto \& Fardella, 2014). En aquel sentido, se concuerda con el materialismo relacional (Soto, Fardella, Valenzuela, \& Carvajal, 2017) pues se concibe a la realidad ante una diversidad de formas materiales que no son casuales, más bien efectos para establecer estratagemas. En rigor, éstas supervisan, gestionan y transforman, originando dinámicas específicas que son precisas indagar situadamente, pues los sujetos se ven interpelados en un espacio interaccional particular. Si extrapolamos este contexto al Trabajo Social chileno, particularmente su relación con la gestión de políticas sociales, es primordial examinar materialidades jurídicas que en más de treinta años han determinado efectos en su quehacer profesional.

Cabe mencionar el Decreto con Fuerza de Ley (DFL) n. ${ }^{\circ} 1$, del 3 de enero de 1981, que específicamente en el art. 12 forja la pérdida del rango universitario. Esta estructuración posibilita su formación en institutos profesionales (IP) que se crean con el DFL n. ${ }^{\circ}$, del 16 de febrero de 1981, precepto que refuerza en aquella época el Trabajo Social oficial vinculado con el cariz funcionalista realizado por profesionales partícipes en la gestión de políticas sociales del gobierno de facto, y por otra, el Trabajo Social alternativo, relacionado con la comprensión y lo crítico, que implica otra expresión profesional en afinidad con la recuperación de la democracia y la defensa de los derechos humanos ${ }^{1}$ (Castañeda \& Salamé, 2012). Como corolario, el carácter eminentemente transformador que propone el Trabajo Social a través de su accionar se conflictúa, y emerge una bifurcación que gradualmente subjetiva su propósito transformador, y que se solidifica un día antes de la recuperación de la democracia con la Ley Orgánica Constitucional de la Enseñanza (LOCE) n. ${ }^{\circ} 18.962$, del 10 de marzo de 1990, que ratifica la pérdida acaecida a inicios de los

\section{La proyección de un}

\section{Trabajador Social como} emprendedor envuelve una nueva composición pues este precepto transporta plusvalía que se concluye en los discursos vinculados con la gestión social, en un anónimo carácter de acción, que no descarta la utilidad económica. 
1980. Entonces se continúa conflictuando su saber y papel en la sociedad, no omitiendo su mercantilización, en un contexto laboral en donde se interpela a la profesión con el fin de erradicar y disminuir la pobreza a través de proyectos sociales propiciados en un estado subsidiario. En el año 2005, tras los esfuerzos de profesionales, académicos y gremio en los decenios de los 1980 y 1990, se produce la recuperación del rango universitario a través de la Ley n. ${ }^{\circ} 20.054^{2}$ (2005), que modifica la LOCE. Sin embargo, con esta materialidad, Trabajo Social puede ser cursado tanto en IP como en universidades privadas y/o públicas.

El grado académico es privativo de las universidades que son las responsables de establecer planes específicos de graduación para los profesionales egresados de institutos. Brota entonces una trifurcación que cosifica a la licenciatura en Trabajo Social, interpelando su domicilio en la investigación social y también su empleabilidad, ante un aumento ostensible en matrículas. En este punto, Castillo y Rodríguez (2016) plantean a la profesión como una de las carreras que más crece en términos de ingreso, por ejemplo, de 5.296 en 1997, para llegar a los 31.460 en el periodo académico 2013. Por lo tanto, las condiciones fundidas en órdenes jurídicas traccionan no solo el ideario de una profesión, sino que las interacciones en la esfera laboral en donde urge ser empleable. La empleabilidad se constituye como exigencia para las personas y se expresa en perfiles legitimados por las políticas sociales, que definen quienes o no participan (Rivera-Aguilera, 2017), en un escenario gradual e inclusive híbrido (Noordegraaf, 2015) que exterioriza tensiones por la flexibilidad laboral (Sisto \& Fardella, 2008), que se extiende a los nuevos mecanismos dirigidos a la gestión en donde es posible suponer performatividades (Briones, 2007) en las profesiones que la transportan.

\section{Interpelaciones semánticas sobre la gestión social}

No hay consenso para comprender a la gestión, sin embargo, en términos latinoamericanos, destaca Ramírez (2016), quien la plantea como un espacio que aporta a la constitución de teorías, conceptos y modos de hacer, esbozando una:

Acción integral, entendida como un proceso de trabajo y organización en el que se coordinan diferentes miradas, perspectivas y esfuerzos, para avanzar eficazmente hacia objetivos asumidos institucionalmente y que desearíamos que fueran adoptados de manera participativa y democrática. (Villamayor y Lamas, 1998 como se cita en Ramírez, 2016, p. 236)

Complementariamente esta autora ubica a la gestión en cuatro campos de conocimiento, el primero corresponde al desarrollo disciplinar del Trabajo Social que cuestiona la realidad social desde bases epistémicas, siendo concordante con lo planteado por Martín Estalayo y Nogués Saéz (2017), quienes esbozan que esta mirada impregna la epistemología del Trabajo Social. El segundo espacio vinculado al desarrollo humano y políticas sociales, enlazado con la consecución de bienestar a través del diseño de políticas sociales. En tercer término, el académico, que plantea a la gestión en el territorio y las redes organizacionales, con el fin de mermar las complejidades que implica la imposición de modelos neoliberales. Por último, la gestión se asocia a la planificación, administración y gerencia en donde es posible situar las influencias del NMP que genera profundas transformaciones en espacios laborales, "involucra un cambio en el modo en que pensamos, actuamos, sentimos; en suma, un cambio en la forma de ser de los individuos y de las organizaciones" (González y Rodríguez, 2014, p. 3 como se cita en Ramírez, 2016, p. 240). En línea con lo distinguido la gerencia aparece como termino subjetivado por el NMP pues el gestor resulta ser un actor fundamental para las políticas con valor en lo público (O’Flynn, 2007). Complementariamente, la gerencia social se orienta al incremento de la efectividad de propuestas de desarrollo social (Mokate \& Saavedra, 2006). Por ende, en lo social hay concordancia entre los mandatos de la gestión y la gerencia, particularmente cuando se enfatiza en la transformación, inclusive Martín Estalayo y Nogués Sáez (2017) plantean que los mandatos y condiciones en la gestión de prestaciones sociales, admiten un derecho subjetivo. Refuerza lo dicho Morales Mosquera (2012) quien bosqueja a la gestión social como prácticas que llevan a cabo diversas organizaciones de la sociedad civil que prestan servicios sociales públicos, orientados a mejorar la calidad de vida de la población en general. En añadidura Mokate y Saavedra (2006) reiteran esta idea, pero entendiendo a la gestión social como práctica estratégica, enfocada en transformaciones sociales, en un contexto en donde se requieren particulares competencias de acción profesional para ser empleable. Por otra parte, versiones académicas en Chile plantean a la gestión o gerencia, como social:

Conformado por la intersección de los ejes recursos, rede y tecnología y conocimiento, información y datos. Es un ámbito de acción profesional contemporáneo, conformado por el conjunto de puestos laborales 
en diferentes organizaciones sociolaborales de acción social propiamente tal y en empresas productivas en que trabajo social es responsable de la gestión de recursos. (Castañeda \& Salamé, 2012, pp. 114-115)

Entonces la gestión social, se asocia a la conducción de planes, programas y proyectos sociales con incidencia pública, en donde destaca el cumplimiento de metas una de las características en la ideología del NMP y del modelo de libre mercado, que alberga otras particularidades:

La matriz ideológica neoliberal no solo ha implicado la expansión de la oferta académica, lo cual es una expresión material de su hegemonía. Sino que por sobre todo, que su peso discursivo, radica en la instalación de un lenguaje que emana desde la elite intelectual, y que no busca sino la producción y reproducción de valores y conductas acorde a sus intereses. (Vivero Arriagada, 2017, p. 348)

Con todo, en este tejido surge la necesidad del análisis de discurso pragmático y retórico propuesto por el grupo de Loughborough (Sisto \& Fardella, 2011) cuyas características fundamentales se relacionan con analizar los textos como formas de acción social. En aquel sentido, resulta preponderante comprender que el discurso corresponde a "un conjunto de prácticas lingüísticas que mantienen y promueven ciertas relaciones sociales” (Iñiguez \& Anaki, 1994, p. 64 como se cita en Garay, Iñiguez, \& Martínez, 2005 , pp. 110-111). Teniendo en cuenta lo anterior, las entrevistas fueron leídas continuamente, para encontrar retoricas que dieran cuenta de versiones que a su vez socaven otras, por ello, el énfasis estuvo en los recursos de factualización, comprendidos como técnicas y mecanismos utilizados por las personas en determinados contextos de interacción. Esta retórica es manejada para construir hechos pudiendo ser utilizados de manera ofensiva o defensiva, en aquel sentido, Potter (1998) da a conocer una variada gama de recursos esgrimida por las personas de manera independiente o fusionada, con el fin de potenciar o atenuar determinadas descripciones de la realidad. Por ende, la tarea se remite a escudriñar por qué es utilizada determinada factualización por los sujetos, con el fin de detectar el núcleo que se busca fortalecer o debilitar, y que no puede omitir el escenario de producción. En base a lo anterior, se constituyen dos ejes discursivos, en primer lugar, el denominado rango universitario, una idealización interpelada por un negocio. En éste se da cuenta del espesor de una de las materialidades que han producido al Trabajo Social desde 2005, arrastrando segmentación y efectos en diversos ámbitos de desempeño laboral. En segundo término, el eje llamado arribo de la individualización, en esta vertiente se da cuenta de un proceso performativo en el ideario del Trabajo Social en donde emerge con propiedad el emprendimiento, etiqueta que certificaría idoneidad en el mercado laboral.

\section{Rango universitario, reivindicación interpelada por un negocio}

Este eje discursivo se sostiene sobre cuatro grandes categorías, la fantasía de la regulación, servicio social on-line, la instalación de fragmentaciones y construyéndose desde la competencia.

a) La fantasía de la regulación.

Se liberaliza la educación superior y eso hace ¿no es cierto? Que exista toda una serie de explosión por decirlo así de programas de formación profesional, donde el trabajo social es una de las carreras que, que explota ¿no es cierto? Pero que finalmente la, si nosotros pensamos que la obtención del rango universitario iba a regular un mercado desregulado de educación superior, no tenía ningún sentido. Es que hoy día la educación es lamentablemente es un negocio ¿ya? (Trabajador Social en gestión social en políticas de vivienda)

En el texto precedente emerge un discurso empirista, que construye versiones de objetos como algo innegable, referido a la mercantilización de la educación superior y del Trabajo Social, coyuntura que plantea a la recuperación del rango universitario como un hito sin sentido, en un escenario de desregulación que desvaloriza a las profesiones. Si complementamos la Ley n. ${ }^{\circ} 20.054$ (2005), la licenciatura se cosifica, es decir, se detalla una conversión de lo abstracto a lo material, al presentarse inclusive como alternativa de formación específica e independiente del título profesional. En complemento, la extensión de matrículas en este caso educativas se conforma epocalmente (du Gay, 2007) es decir, se emplea la dicotomía y alternativas de acción entre temporalidades pasadas y presentes en el mercado laboral, para plantear el negocio del trabajo social en educación superior. 
b) Servicio social on-line.

Bueno yo creo que hay una oferta pa todo, pero indudablemente hay una oferta eh amplia y, y, y... y con mucho marketing detrás ¿no es cierto? Eh... del servicio social, o sea, yo el otro día pensaba que no era cierto, pero, me di cuenta que había gente que podía estudiar servicio social online . . . cómo salen profesional de ahí y qué es lo que hacen digamos eh o qué tipos de profesional salen de ahí. (Trabajador Social en gestión social en políticas educativas)

Se observa una interpelación verbal a la ideología del NMP pues hay una retórica ofensiva, es decir, una relación antagónica en términos semánticos, pues se socava la noción de oferta y que se extiende también al de marketing. Ambos términos, resultan subjetivados por el hablante, quien los cosifica cuando se relacionan con la plataforma formativa on-line en Trabajo Social. Complementariamente y respecto a los egresados en este contorno, brota una acreditación de categorías, que consiste en atestiguar la pertenencia de un sujeto a una condición social para socavar, pues se cuestiona la validez de estos actores ya que se interpreta sospecha en un despliegue laboral.

c) La instalación de las fragmentaciones.

"Que estamos altamente eh... fraccionados, divididos, tensionados en cómo miramos el trabajo social" (Trabajador Social en gestión social en políticas educativas).

De acuerdo con un discurso empirista se demuestra la entelequia del núcleo común en Trabajo Social, pues se presentan segmentaciones que intiman a la profesión, que, sumado a una factualización de consenso, consistente en demostrar que una aseveración no viene de un grupo reducido, sino que amplio, se afirma una tensión respecto a la autobservación de la profesión. La fragmentación entonces aparece cosificada y en esta es posible interpretar incertidumbres para el Trabajo Social. Si asociamos aquello a lo señalado por Sisto y Fardella (2008), los Trabajadores Sociales no se encontrarían determinados y sujetos a nada, siendo libres electores en su devenir.

d) Construyéndose desde la competencia.

Dan trabajo al trabajador social, pero tienen que ser muy competitivos ¿ya? Y ahí, a eso agregamos el factor político. Entonces hoy día yo creo que el profesional que es competitivo, que se perfecciona eh... puede acceder a ciertos puestos de trabajo significativos ¿ya? (Trabajador Social en gestión social en políticas de vivienda)

En el texto la competitividad se erige como una acreditación de categoría para construirse como un sujeto empleable, consideración que nos conecta con el trabajo de Sisto (2009), quien establece que las competencias que se necesitan son aquellas que el mercado laboral prescribe para emplearse. Para el autor en los escenarios laborales lo que importa es la transferibilidad de competencias, por ende, el sujeto debe hacerse a sí mismo, por ejemplo, a través de sucesivos perfeccionamientos (Saldaña, 2008). Entonces la competencia se cosifica en un discurso epocalista en el mercado laboral, es más, Freidson (2001) señala que esta profesión está siendo redefinida en su relación con el estado, pues la economía capitalista progresivamente está cimentando una ruta para consolidar proveedores privados.

\section{El arribo de la individualización en Trabajo Social}

El presente eje está compuesto por cuatro grandes categorías entre las que destacan, rentabilizar lo social, mercadear y contratar, fundando proyectos propios y el emprendimiento.

a) Rentabilizar lo social.

Poder rentabilizar el producto social te implica la intervención del trabajo social con otra mirada, o sea, mi mirada es social pero también mi mirada es comercial ¿ya? Y en ese contexto he desarrollado estructuras de negocio en donde se rentabiliza lo social. O sea, el asistente social no tiene porque no ganar plata, también tiene que ganar plata ¿ya? Pero obviamente con una vocación social. (Trabajador Social en gestión social en políticas educativas) 
Saldaña (2008) plantea en el contexto del NMP el advenimiento de una nueva terminología, basada en tropos administrativos que emergen como recursos modalizadores, es decir, que modifican, para reforzar una subjetividad mercantil cosificando términos tales como rentabilización, estructuras de negocio, mirada comercial, y ganar plata. En el extracto se compone una gestión de intereses, recurso asociado a una construcción lingüística en la que se denota indolencia, pero a la vez se resalta un interés a través de versiones alternativas. En este contexto se pactan dos fines, por un lado, obtener dinero y por otro, vocación social. Esta contradicción potencialmente tensiona la ética de lo público (Fardella, Sisto, Morales, Rivera, \& Soto, 2016) realidad sobre la que es dable preguntarse por nuevas explicaciones en cuanto a la transformación que propicia el Trabajo Social.

\section{b) Mercadear y contratar.}

El producto social también tiene que rentar, rentabilizarse, si también tiene que comercializar y con eso tú también podí generar fuente de trabajo para otros profesionales, que ha sido mi caso, acá yo trabajo con arquitectos, con constructores, con abogados, con asistentes sociales iya? Y, y, y yo soy trabajador social, es que estoy a cargo del tema, entonces ¿por qué soy trabajador social? Porque he tenido la experiencia de la dirección o de la coordinación o también he tenido la inquietud ¿no cierto? (Trabajador Social en gestión social en políticas de vivienda)

En el texto es factible la empleabilidad si se interconectan rentabilidad y producto social, ambos términos conforman una provisión de detalles, vale decir, se provee de caracterizaciones para que la frase e idea parezca factual, haciendo notar experiencia, en términos de gestión. Por otra parte, en conformidad al recurso lingüístico de posicionamiento, que corresponde a un recurso dinámico cuyo centro consiste en mostrarse cercano o distanciado de una afirmación, es posible interpretar a un Trabajador Social como responsable de lo gerencial. Pero, éste se escinde del equipo profesional, fundándose como experto en la dirección y coordinación, entonces su relato se factualiza conforme una gestión de la conveniencia (Potter, 1998).

\section{c) Fundando proyectos propios.}

Mira, lo mismo, lo mismo, yo creo que la capacidad de, de, de poder generar eh proyectos propios como profesional, hacer ejercicio libre de la profesión tiene que ver con que tú tengas un nivel eh... de manejo de los temas que son propios de la, de, de, de nuestra especialidad, ya, y estoy hablando no sé po' ... de política, de vivienda, de adulto mayor, eh... de infancia, de lo que sea, ya, que tenga cierta profundidad y donde va esto, si yo eh... hablando de empleabilidad por ejemplo, voy y te propongo un, un proyecto a un empleador o a un financista o a un inversor, pongámosle en nombre que quieras ya. (Trabajador Social en gestión social en políticas educativas)

En el texto el hablante de acuerdo a un discurso empirista establece que el ejercicio libre de la profesión, a través de proyectos sociales, depende de los conocimientos que se tengan respecto a determinadas áreas. En términos de empleabilidad estas condiciones son factibles cuando se presenta a un inversor, por tanto, un Trabajador Social diligente debe hacerlo conforme a los criterios de las empresas, y en el mercado (Saldaña, 2008). La retórica defensiva que se usa para resistir socavamientos plantea una estructura en la que se presentan dos actores fundamentales para desplegar la fundación de proyectos individuales, por un lado, el profesional y por otro, el financista reificando un carácter gerencial en estas relaciones laborales.

d) El emprendimiento.

Yo creo que a los a los nuevos estudiantes, claro, o sea genérense nuevas oportunidades, ustedes mismos, ¿ya? En nivel de emprendimiento, eh... en el nivel de desarrollo de proyectos, o sea ¿Me entiendes? Para que ellos mismos se busquen su espacio, porque hoy día frente a la saturación del mercado laboral eh... y es frustrante, es penoso ver que estamos pasando con los profesionales jóvenes. (Trabajador Social en gestión social en políticas de vivienda)

El discurso inscribe a un sujeto como responsable en el desarrollo de sus oportunidades, individualizando su posición en el mercado laboral, apareciendo el emprendimiento como garantía de acreditación en lo laboral $\mathrm{y}$ en el desarrollo de proyectos. De manera complementaria el enunciado exterioriza particularidades epocalistas (du Gay, 2007), destacando el rebose de profesionales, especialmente jóvenes. Frente a lo anterior, la retórica 
del emprendimiento se domicilia como bisagra en una jerarquía de modalización, recurso en el que se denota un proceso de cosificación que intenta hacer que una descripción ascienda en jerarquía, y con otra, mediante ironías u otros artificios hacerla descender. En este sentido lo modalizado consiste en emplearse o no en el mercado laboral, refrendando lo dicho por Humphrey (2003) respecto a que el trabajo social ha sido reformado y regulado ostensiblemente en cuanto a sus modos de actuación. En esta línea Leigh (2014) establece que la fuerza del mercado ha impactado notablemente al Trabajo Social, especialmente en sus prácticas de implementación con sujetos y colectivos.

\section{Conclusiones}

Los resultados en este escrito son situados, y cabe interrogarse por la anhelada transformación del Trabajo Social que se instruye en aulas y desarrolla en su práctica, en un ámbito de desempeño que lo interpela. La reflexión da cuenta de la complejidad del NMP, perspectiva que se inscribe subyacentemente en el accionar de las profesiones, en una diversidad de espacios laborales en donde es factible especular sobre un Trabajo Social subjetivado. Para aquello se considera esencial el seguimiento a materialidades y discursos que prescriben un ejercicio performado e inclusive iconográfico, cuando se analiza a las carreras profesionales signadas por la densidad mercantil, que desprofesionalizan indivisiblemente. Otorga vigor a lo antes dicho, la reflexión concerniente al primer eje discursivo, en donde emerge la factualización de una rotura en un ideario profesional, ante una lógica managerial que escamotea la voluntad de un colectivo profesional, convergiendo una solución afín al mercado que perpetua y fragmenta al Trabajo Social en este siglo.

Este fraccionamiento envuelve efectos en términos identitario laborales (Sisto, 2012), pues ante una triple plataforma que cosifica a la licenciatura, se dificulta en Chile el esclarecimiento sobre el real depositario histórico del Trabajo Social. Esta coyuntura funda interpelaciones en los discursos de gestión social, que se forjan originariamente en materialidades que imprimen individualismo y disciplinamiento, normalizando el peculio y tensionando la ética de lo público. En este hilo conductor, es dable preguntarse por cambios en la Ley n. ${ }^{\circ}$ 20.054 (2005), pues da fuerza a la distancia vigente en las versiones disciplinares y profesionales en Trabajo Social, que se fortalecen por el exuberante número de egresados, afirmando respecto a su reconocimiento, una fantasía no cumplida. Por otra parte, situados en la traducción narrativa del segundo de los ejes discursivos vinculado con el emprendimiento, éste nos aproxima a una nueva identidad para sí y otros (Dubar, 2000).

En aquel sentido, la proyección de un Trabajador Social como emprendedor envuelve una nueva composición pues este precepto transporta plusvalía que se concluye en los discursos vinculados con la gestión social, en un anónimo carácter de acción, que no descarta la utilidad económica. En consecuencia, con un acoplamiento managerial se forja un relato identitario laboral que da cuenta de un sujeto que es capaz de ejecutar negocios, alternativas de empleo y crear empresas, por añadidura, su desarrollo depende exclusivamente de él, tanto el éxito como el fracaso. Entonces, cabe interrogarse por la narrativa del agente de cambio, que alberga las más profundas metamorfosis de las estructuras sociales que oprimen. Por ende, es dable imaginarse una jerarquía de modalización (Potter, 1998) entre el gestor-emprendedor y el agente-cambio en los escenarios laborales. Consecuentemente, cabe conflictuar la nueva gestión social transformadora, subjetivada por el NMP que requiere de nuevas traducciones para proyectar no solo comprensión y cambio en otros, sino que sobre sí. Empero, el Trabajo Social debe vislumbrar que las herramientas de gestión que utiliza son discursos que tienen soportes ideológicos y estéticos que pueden ser desmontados por los estudios críticos del management, que no suprime gestiones con orientación transformadora, solo que, desde un plano de micro transformaciones. Esta nueva esencia puede imprimir una innovada versatilidad al Trabajo Social, para así conflictuar la estructuración del NMP, construyendo dialécticamente una nueva subjetividad como parte integrante de las fuerzas del trabajo tanto en el presente como en el futuro.

\section{Referencias}

Briones, C. (2007). Teorías performativas de la identidad y performatividad de las teorías. Tabula Rasa, 6, 55-83.

Castañeda, P., \& Salamé, A. (2012). Profesionalidad del Trabajo Social chileno: Tradición y transformación. Temuco, Chile: Universidad de La Frontera.

Castillo, V., \& Rodríguez, C. (2016). Los problemas del stock en campos profesionales difusos: Oferta educativa en Trabajo Social. Estudios Pedagógicos, 42(1), 37-52. doi:10.4067/S0718-07052016000100003

Decreto con Fuerza de Ley n. ${ }^{\circ} 1$ art. 12, Enero 3, 1981, Fija normas sobre universidades, Diario Oficial [D.O.]. Recuperado de https:/ /www.leychile.cl/Navegar?idNorma=3394\&idParte $=7112349$ 
Decreto con Fuerza de Ley n. ${ }^{\circ}$ 5, Febrero 16, 1981, Fija normas sobre institutos profesionales, Diario Oficial [D.O.]. Recuperado de https://www.leychile.cl/Navegar?idNorma=3668

Dictamen n. ${ }^{\circ}$ 39296, Mayo 15, 2015, Contraloría General de la República [C.G.R.]. Recuperado de https://www.contraloria.cl/pdfbuscador/ dictamenes/039296N15/html

Dubar, C. (2000). La crise des identités : L'interprétation d'une mutation. Paris, Francia: Presses Universitaires de France.

du Gay, P. (2007). Organizing identity: Persons and organizations 'after theory'. London, Reino Unido: Sage.

Fardella, C., Sisto, V., Morales, K., Rivera, G., \& Soto, R. (2016). Identidades laborales y ética del trabajo público en tiempos de rendición de cuentas. Psykhe, 25(2), 1-11. doi:10.7764/psykhe.25.2.789

Freidson, E. (2001). La teoría de las profesiones. Estado del arte (L. Godinas \& J. Armendáriz Pérez, Trans.). Perfiles Educativos, 23(93), 28-43.

Garay, A., Iñiguez, L., \& Martínez, L. (2005). La perspectiva discursiva en psicología social. Subjetividad y Procesos Cognitivos, 7 , 105-130.

González-Rey, F., \& Mitjáns, A. (2016). Una epistemología para el estudio de la subjetividad: Sus implicaciones metodológicas. Psicoperspectivas, 15(1), 5-16. doi:10.5027/psicoperspectivas-Vol15-Issue1-fulltext-667

Grassi, E. (2009). La normalidad del trabajo, empleos normales y vidas de trabajo: razones para trabajar. Revista Katálysis, 12, $226-234$. doi:10.1590/S1414-49802009000200012

Humphrey, J. C. (2003). New Labour and the regulatory reform of social care. Critical Social Policy, 23, 5-24. doi:10.1177/ 026101830302300101

Leigh, J. T. (2014). The process of professionalization: Exploring the identities of child protection social workers. Journal of Social Work, 14, 625-644. doi:10.1177/1468017313504380

Ley n. ${ }^{\circ}$ 18962, Marzo 10, 1990, Ley Organica Constitucional de Enseñanza, Diario Oficial [D.O.]. Recuperado de https://www.leychile.cl/ Navegar?idNorma $=30330$

Ley n. ${ }^{\circ} 20054$, Septiembre 27, 2005, Modifica la Ley n ${ }^{\circ}$ 18.962, Organica Constitucional de Enseñanza, restableciendo la exclusividad universitaria del trabajo social, Diario Oficial [D.O.]. Recuperado de https://www.leychile.cl/Navegar?idNorma=242374

Martín Estalayo, M., \& Nogués Sáez, L. (2017). El trabajo social en los aparatos del Estado y su posicionamiento ético-político en la garantía de los derechos sociales. Revista Katálysis, 20,335-343. doi:10.1590/1982-02592017v20n3p335

Mokate, K., \& Saavedra, J. (2006, marzo). Gerencia social: Un enfoque integral en la gestión de políticas y programas (Documentos de Trabajo del INDES, I-56). Recuperado de https://publications.iadb.org/publications/spanish/document/Gerencia-Social-Un-EnfoqueIntegral-para-la-Gesti\%C3\%B3n-de-Pol\%C3\%ADticas-y-Programas.pdf

Morales Mosquera, M. (2012). Los estudios organizacionales: Un complemento para el estudio de la gestión social. AD-minister, 21, 117-133.

Noordegraaf, M. (2015). Hybrid professionalism and beyond: (New) Forms of public professionalism in changing organizational and societal contexts. Journal of Professions and Organization, 2, 187-206. doi:10.1093/jpo/jov002

O’Flynn, J. (2007). From new public management to public value: Paradigmatic change and managerial implications. Australian Journal of Public Administration, 66, 353-366. doi:10.1111/j.1467-8500.2007.00545.x

Potter, J. (1998). La representación de la realidad. Buenos Aires, Argentina: Paidós.

Potter, J., \& Wetherell, M. (1987). Discourse and social psychology: Beyond attitudes and behaviour. London, Reino Unido: Sage.

Ramírez, C. (2016). Tendencias investigativas que delimitan los campos de actuación del trabajo social en la gestión social. Civilizar Ciencias Sociales y Humanas, 16(30), 233-244.

Rivera-Aguilera, G. (2017). Los procesos de influencia global/local en políticas públicas: Una propuesta metodológica. Psicoperspectivas, 16(3), 110-120. doi:10.5027/psicoperspectivas-Vol16-Issue3-fulltext-1092

Saldaña, A. (2008). La sociedad enferma de gestión. Ideología gestionaria, poder managerial y hostigamiento social. CPU-e, Revista de Investigación Educativa, 7, 1-6.

Sisto, V. (2009). Cambios en el trabajo, identidad e inclusión social en Chile: Desafíos para la investigación. Revista Universum, 24(2), 192-216. doi:10.4067/S0718-23762009000200011

Sisto, V. (2012). Identidades desafiadas: Individualización, managerialismo y trabajo docente en el Chile actual. Psykhe, 21(2), 35-46. doi: 10.7764 psykhe.21.2.542

Sisto, V., \& Fardella, C. (2008). Narrándose en la flexibilidad. Un análisis narrativo discursivo de la identidad en tiempos de flexibilidad laboral. Revista de Psicología, 17(2), 59-80.

Sisto, V., \& Fardella, C. (2011). Nuevas políticas públicas, epocalismo e identidad: El caso de las políticas orientadas a los docentes en Chile. REU - Revista de Estudos Universitários, 37(1), 123-141.

Sisto, V., \& Fardella, C. (2014). El eclipse del profesionalismo en la era de la rendición de cuentas - modelando docentes en el contexto del Nuevo Management Público. Cadernos de Educação, 49, 3-23.

Soto, Á., Fardella, C., Valenzuela, A., \& Carvajal, F. (2017). Las funciones performativas de los instrumentos en las redes de acción pública. Psicoperspectivas, 16(3), 76-86. doi:10.5027/psicoperspectivas-Vol16-Issue3-fulltext-999

Vivero Arriagada, L. (2017). Desafíos de una práctica ético-política. El trabajo social chileno post-dictadura. Revista Katálysis, $20,344-$ 352. doi:10.1590/1982-02592017v20n3p344 


\section{Notas}

1 Las autoras desarrollan diferentes transiciones en la historia del trabajo social en el siglo XX, desde sus inicios en 1925, hasta el presente, caracterizando a este tiempo por variadas transformaciones en las tradiciones profesionales.

2 Esta Ley, junto con el Dictamen n. ${ }^{\circ}$ 39.296, del 15 de mayo de 2015, terminan por clausurar la trifurcación de la profesión, a saber, Trabajo o Servicio Social con licenciatura, sin licenciatura y con licenciatura tardía.

\section{Raúl Octavio Hozven Valenzuela}

raul.hozven.v@hotmail.com

Máster en Educación por la Pontificia Universidad Católica de Valparaíso (PUCV)

Jefe de Carrera de la Escuela de Trabajo Social de la Universidad Santo Tomás (UST) en Viña del Mar

\section{UST}

Avda. Limonares, 190

Viña del Mar - Región de Valparaíso - Chile

CP: 2561780

\section{Agencia financiadora}

Investigación financiada por la Universidad Santo Tomás (UST), Chile. Fondo regular de proyectos de investigación internos. Proyecto n. ${ }^{\circ} 22721$.

Período de ejecución: enero de 2016 a diciembre de 2017.

\section{Contribuciones de los autores}

No se aplica.

Aprobación por Comisión de Ética y consentimiento para participación

Aprobado por el Comité Ético Científico (CEC) UST, No 160/

2015, 27 de diciembre de 2015. A cada profesional se aplicó un consentimiento informado, y se firmaron dos copias, con miras a resguardar su dignidad, confidencialidad y anonimato.

\section{Consentimiento para publicación}

No se aplica (porque los datos personales de los encuestados no son revelados).

\section{Conflicto de intereses}

No hay conflicto de intereses. 